\title{
Changes in Myocardial Perfusion Reserve After PTCA: Noninvasive Assessment with Positron Tomography
}

\begin{abstract}
Richard A. Goldstein, Richard L. Kirkeeide, Richard W. Smalling, Akira Nishikawa, Michael E. Merhige, Linda L. Demer, Nizar A. Mullani, and K. Lance Gould

Positron Diagnostic and Research Center and Cardiology Division, University of Texas Medical School at Houston, Texas

The effect of percutaneous transluminal coronary angioplasty (PTCA) on myocardial perfusion reserve has not been previously determined. Accordingly, 11 patients underwent positron imaging with $\left[{ }^{13} \mathrm{~N}\right]$ ammonia or ${ }^{82} \mathrm{Rb}$ at rest and following dipyridamole + handgrip stress before and after PTCA. The ratio of stress to rest activity (S:R) was determined for each region of interest. Relative myocardial perfusion reserve by positron tomography (RMPR) was calculated by dividing $S: R$ of the stenotic area by a corresponding value from a normal reference area of the same patient. Automated quantitative coronary arteriography was used to objectively measure the percent diameter (\%D) and the percent area narrowing (\%A) of the stenoses. In nine patients with successful PTCA, \%D and \%A improved $68 \pm 10$ to $49 \pm$ $15 \%$ and $92 \pm 3$ to $72 \pm 5 \%$ ) and RMPR increased from $0.79 \pm 0.07$ to $0.96 \pm 0.05$. In the two patients in whom PTCA was unsuccessful, RMPR was unchanged. Changes in RMPR correlated inversely with changes in $\% D(r=-0.68)$ and $\% A(r=-0.92)$ and directly with improved coronary flow reserve derived from all stenosis measurements $(r=0.73, p<0.001$ for each). This study suggests that dipyridamole + handgrip stress imaging with PET can be used to assess changes in myocardial pertusion reserve before and after PTCA with the potential for determining restenosis noninvasively.
\end{abstract}

J Nucl Med 28:1262-1267, 1987

$P_{\text {eto }}$ major therapeutic advance for the treatment of coronary artery disease. This technique is less expensive than coronary artery bypass surgery and patients can usually resume normal activities within a few days after the procedure. However, important questions remain about selection and follow-up of these patients. The decision to perform angioplasty is usually based on the severity of the stenosis and symptoms. Percent arterial narrowing from coronary arteriograms may not accurately reflect the significance of a stenosis because it does not account for other important critical measures of the stenosis such as the length and absolute crosssectional luminal area of the involved segment $(1,2)$. Symptoms of chest pain may be unreliable indicators because of variability in the perception of pain by

Received Sept. 8, 1986; revision accepted Feb. 23, 1987.

For reprints contact: Richard A. Goldstein, MD, Nuclear Cardiology, Cardiology Division, The University of Texas Medical School, P.O. Box 20708, Rm. 1.246, Houston, TX 77225. patients and the effect of cardiac drugs. Noninvasive tests such as exercise thallium scintigraphy can be used to qualitatively assess perfusion abnormalities, but the physical properties of thallium-201 and other single photon emitters preclude absolute quantitation of regional perfusion (3).

Positron emission tracers have decay characteristics that permit measurement of the uptake of tracers such as rubidium-82 $\left({ }^{82} \mathrm{Rb}\right)$ and nitrogen-13 $\left({ }^{13} \mathrm{~N}\right)$ ammonia to quantitatively assess regional perfusion (4-7). Accordingly, the purpose of the current study is to determine the relation between changes in relative myocardial perfusion reserve by positron emission tomography, and coronary anatomy after percutaneous transluminal coronary angioplasty.

\section{PATIENTS AND METHODS}

\section{Patient Population}

Eleven patients (seven men, four women), underwent coronary arteriography and positron imaging before and after 
percutaneous transluminal coronary angioplasty. The patients ranged in age from 34 to $62 \mathrm{yr}$ [mean $=52.0 \pm 8.8 \mathrm{yr}$ (s.d.)]. Angioplasty was performed on the left anterior descending coronary artery in eight patients, the left circumflex in two and an obtuse marginal branch in one. Patients with unstable angina or myocardial infarction were excluded from the study. Patients were imaged within 1 wk before their coronary angioplasty and an average of 11.5 days afterwards. The study protocol was approved by the University of Texas Medical School at Houston Committee for the Protection of Human Subjects, and all patients gave informed consent prior to entry into the study.

Coronary arteriography was performed using either the Judkins or Sones technique. Multiple coronary angiograms were obtained using 3-10 $c$ of Hypaque-76 per injection with a biplane 5-7-9 in. image intensifier. "The $x$-ray tube ${ }^{\dagger}$ had a 0.6-1.0 mm focal spot operating at 4-6 msec exposures with $300 \mathrm{~mA}$ and $109 \mathrm{kV}$. Low contrast Vari-Cath cine film was used at 30-60 frames/s. Orthogonal views were obtained for quantitative analysis of the angiograms. The cine system resolution was 4-5 line pairs/mm. Pin cushion distortion and variation of magnification in different parts of the field were $<10 \%$ except at the corners of the field but were corrected from measurements of an imaged grid whose dimensions were known (1).

\section{Analysis of Coronary Angiograms}

Stenosis of major proximal coronary arteries were quantitatively analyzed as previously described (1). Briefly, enddiastolic frames from orthogonally paired angiographic views of each stenosis were optically magnified, digitized, and corrected for the point spread function of the $x$-ray system providing a final spatial resolution of $\pm 0.1 \mathrm{~mm} / \mathrm{pixel}$. After selecting the arterial segment of interest, the images were analyzed by an automated arterial border recognition program and cross-sectional densitometry from which vessel diameters and cross-sectional areas were determined. Spatial calibration was determined using the catheter as a size reference. Specific anatomic characteristics of each stenosis, including minimal cross-sectional area, relative percent narrowing (diameter and area), and integrated length effects were determined. Pressureflow characteristics of the stenotic segment were then derived by computing the viscous and expansion coefficients of pressure loss which were used to determine the coronary stenosis flow reserve (CFR) as previously validated (1). In this paper, percent narrowing was reported as the most severe stenosis from orthogonal views of the vessel undergoing angioplasty. Dimensional measurements by this method have been previously shown to be accurate to within $\pm 0.1 \mathrm{~mm}$ in $x$-ray phantoms of coronary arteries $(0.5-5.0 \mathrm{~mm}$ in diameter) in scattering media, and reproducible to within $2-3 \%(8,9)$. In dog experiments, these methods correctly predicted measured values to within 0.5 CFR units over a wide range of conditions (1).

\section{Positron Imaging Protocol}

Patients were fasted for at least $4 \mathrm{hr}$ prior to the study and drinks or drugs containing theophylline or caffeine were withheld for at least $\mathbf{8 ~ h r}$ to avoid antagonizing the vasodilatory effects of dipyridamole. Chest fluoroscopy was performed to mark the inferior border of the heart and patients were then positioned in the University of Texas positron camera. The position was marked with a laser light source. A transmission scan (200 million counts) was performed using a Plexiglas ring containing $3 \mathrm{mCi}$ of gallium- 68 . The ring was then removed, the patient's position was re-checked and a resting emission scan was obtained by intravenous injection of either ${ }^{82} \mathbf{R b}$ ( $n$ $=2$, mean dose $=36.7 \pm 5.2 \mathrm{mCi}$ or $\left[{ }^{13} \mathrm{~N}\right]$ ammonia $(\mathrm{n}=9$, mean dose $=16.5 \pm 1.7 \mathrm{mCi}$ ) in an ungated mode without time-of-flight correction as previously described (10). For ${ }^{82} \mathrm{Rb}$, acquisition of emission images was started $1 \mathrm{~min}$ after the end of infusion to minimize blood-pool activity. Data acquisition was stopped 5-7 min later. For $\left[{ }^{13} \mathrm{~N}\right]$ ammonia, acquisition of images was started 3 min after injection and continued for $15 \mathrm{~min}$. After an appropriate delay to allow for decay of counts from the resting study, the same tracer was injected during stress induced by dipyridamole plus handgrip stress. Patients were given a $0.142 \mathrm{mg} / \mathrm{kg} / \mathrm{min}$ i.v. infusion of dipyridamole for $4 \mathrm{~min}$ (total dose $0.568 \mathrm{mg} / \mathrm{kg}$ ). The intravenous line was then flushed with normal saline and electrocardiograms were recorded. Four minutes after the dipyridamole infusion was completed, handgrip was performed by the patient using a mechanical spring device at $25 \%$ of maximal strength and maintained for $4 \mathrm{~min}$. Two minutes into handgrip, the tracer was injected and emission images were acquired in a manner identical to the resting study. Particular attention was paid to positioning of the patient in the same location as in the rest scan.

\section{Analysis of Positron Images}

Nine transaxial slices of the rest and stress studies were displayed in color on a CRT monitor. Regions of interest in the lateral, anterior, septal, and posterior left ventricular walls were defined using an interactive software program that recorded the mean activity, number of pixels, and standard deviation of the activity. A histogram of the activity in each region of interest was also obtained to minimize inclusion of admixtures of normal and abnormal tissue. Mean activity in each region had a standard deviation under $10 \%$ of mean activity for that region. If a stress defect was present, average counts were quantified for each slice on which it was evident. Average activity for each region was calculated for resting and stress images. Relative myocardial perfusion reserve (RMPR) by positron tomography was calculated by the following equation:

$$
\mathbf{R M P R}=\frac{\mathrm{S} / \mathrm{R} \text { stenosis }}{\mathrm{S} / \mathrm{R} \text { normal }},
$$

where $S$ and $\mathbf{R}$ represent regional activity during stress (dipyridamole plus handgrip) and rest, respectively. This value therefore represents the fraction of normal myocardial perfusion reserve within the region supplied by the artery undergoing angioplasty. Although not an absolute measure of perfusion reserve per se, this approach avoids the necessity of obtaining arterial samples to measure the arterial input function of the tracer. The theoretical basis of this measurement has previously been described (11). The normal region was verified by comparison to the arteriogram.

\section{Statistical Amalysis}

The relations between changes in relative myocardial perfusion reserve and changes in quantitative arteriographic measures of stenosis severity were determined using a linear best fit to a least squares equation. 


\section{RESULTS}

\section{Effects of PTCA on Stenosis Anatomy and Relative Myocardial Perfusion Reserve}

The effects of angioplasty on geometric stenosis dimensions and relative myocardial perfusion reserve are presented in Table 1. Nine patients had improvement in anatomy, relative myocardial perfusion reserve and a reduction in the transstenotic pressure gradient after angioplasty. In Patients 6 and 8 PTCA was not successful by either stenosis anatomy or relative myocardial perfusion reserve. In Patient 8 , the artery dissected after the initial crossing with the guide wire and the procedure was aborted. The other case (Patient 6) was felt to be successful at the time of angioplasty based on visual improvement of the stenosis and a $35 \mathrm{mmHg}$ reduction in the mean pressure gradient across the stenosis. However, neither quantitative analysis of the arteriograms nor perfusion data from positron tomography demonstrated any significant change.

\section{Relation of Changes in Stenosis Dimensions to Changes in Relative Myocardial Perfusion Reserve After Angioplasty}

Changes in stenosis geometry were associated with changes in relative myocardial perfusion reserve (Fig. 1). In the nine patients that had arteriographic improvement after angioplasty, percent diameter narrowing decreased from $68 \pm 10$ to $49 \pm 15 \%$ and percent area narrowing was reduced from $92 \pm 3$ to $72 \pm 5 \%$. Coronary flow reserve, based on all stenosis measurements, increased from $1.4 \pm 0.4$ to $3.4 \pm 0.6$. Mean relative myocardial perfusion reserve in these patients improved from $0.79 \pm 0.07$ to $0.96 \pm 0.05$. In contrast, in the two patients without a significant change in anatomic stenosis severity, there was no change in relative myocardial perfusion reserve.
Figures 2 and 3 relate the changes in percent diameter and percent area narrowing of the stenosis to changes in relative myocardial perfusion reserve by positron tomography. Reductions in percent diameter narrowing were inversely related to increases in relative myocardial perfusion reserve $(r=-0.68)$. The relation between changes in percent area narrowing and changes in relative myocardial perfusion reserve after angioplasty were similar, but with less scatter and a higher correlation coefficient, -0.92 . Changes in absolute minimal cross-sectional were not closely related to changes in relative myocardial perfusion reserve with an $r=0.52$, p $>0.10$. Improvement in arteriographically determined coronary flow reserve was directly related to increases in relative myocardial perfusion reserve by positron tomography $(r=0.73$, Fig. 4).

\section{DISCUSSION}

When coronary lesions are suitable, PTCA is generally utilized for proximal stenosis of $>60 \%$ diameter narrowing. The criteria for successful PTCA according to the NIH registry for coronary angioplasty has been a $>\mathbf{2 0 \%}$ increase in luminal diameter following the procedure (12). However, visual determination of percent narrowing is highly variable and coronary lesions are often eccentric $(1,2)$. Recent studies have suggested that percent diameter narrowing may not accurately reflect coronary flow reserve since flow is affected by other dimensions such as length and absolute radius (1-3). Accordingly, several investigators have suggested other criteria for successful angioplasty that rely on indirect measures of perfusion reserve (13-18).

In the current study, positron emission tomography was performed before and after PTCA to obtain a more direct measure of the physiological effects of coronary

TABLE 1

Quantitative Arteriographic and Positron Imaging Data Before and After Angioplasty

\begin{tabular}{|c|c|c|c|c|c|c|c|c|c|c|c|c|c|}
\hline \multirow{2}{*}{$\begin{array}{c}\text { Patient } \\
\text { no. }\end{array}$} & \multirow[b]{2}{*}{ Artery } & \multicolumn{2}{|c|}{ MPR } & \multicolumn{2}{|c|}{$\% D$} & \multicolumn{2}{|c|}{$\% A$} & \multicolumn{2}{|c|}{ CFR } & \multicolumn{2}{|c|}{ Min area } & \multicolumn{2}{|c|}{$\Delta P$} \\
\hline & & Pre & Post & Pre & Post & Pre & Post & Pre & Post & Pre & Post & Pre & Post \\
\hline 1 & LAD & 0.70 & 0.86 & 68 & 51 & 90 & 73 & 1.7 & 3.4 & 0.6 & 1.7 & 58 & 20 \\
\hline 2 & LAD & 0.86 & 1.00 & 66 & 54 & 94 & 73 & 1.2 & 3.7 & 0.9 & 2.7 & 25 & 18 \\
\hline 3 & LAD & 0.77 & 0.95 & 78 & 59 & 95 & 77 & 0.9 & 2.6 & 0.4 & 2.1 & 78 & 25 \\
\hline 4 & LAD & 0.75 & 1.00 & 74 & 21 & 90 & 62 & 1.8 & 4.3 & 0.5 & 3.0 & 80 & 15 \\
\hline 5 & LAD & 0.74 & 1.00 & 74 & 53 & 91 & 67 & 1.4 & 4.0 & 0.3 & 1.0 & 33 & 13 \\
\hline 6 & LAD & 0.89 & 0.80 & 67 & 62 & 75 & 77 & 3.4 & 3.2 & 0.6 & 0.9 & 45 & 10 \\
\hline 7 & LCX & 0.76 & 0.93 & 78 & 55 & 93 & 74 & 1.2 & 3.5 & 0.6 & 2.4 & 34 & 8 \\
\hline 8 & LCX & 0.79 & 0.79 & 78 & 80 & 95 & 96 & 0.9 & 0.7 & 0.4 & 0.4 & - & - \\
\hline 9 & LAD & 0.82 & 0.96 & 69 & 70 & 89 & 77 & 1.6 & 3.1 & 1.1 & 1.5 & 50 & 20 \\
\hline 10 & LAD & 0.91 & 0.97 & 62 & 50 & 87 & 72 & 2.2 & 2.5 & 0.9 & 1.3 & 29 & 9 \\
\hline 11 & OM & 0.83 & 1.00 & 46 & 28 & 95 & 76 & 0.9 & 3.4 & 0.2 & 1.7 & 50 & 27 \\
\hline
\end{tabular}

- MPR = Relative myocardial perfusion reserve; \%D = Percent diameter narrowing; \%A = Percent area narrowing; CFR = Coronary flow reserve arteriographically determined for all stenosis dimensions; $m$ in area $=$ Minimal cross-sectional lumen area; $\Delta P=T r a n s t e n o t i c$ pressure gradient; LAD = Left anterior descending coronary artery; LCX = Left circumflex coronary artery; OM = 1st obtuse marginal coronary artery. 

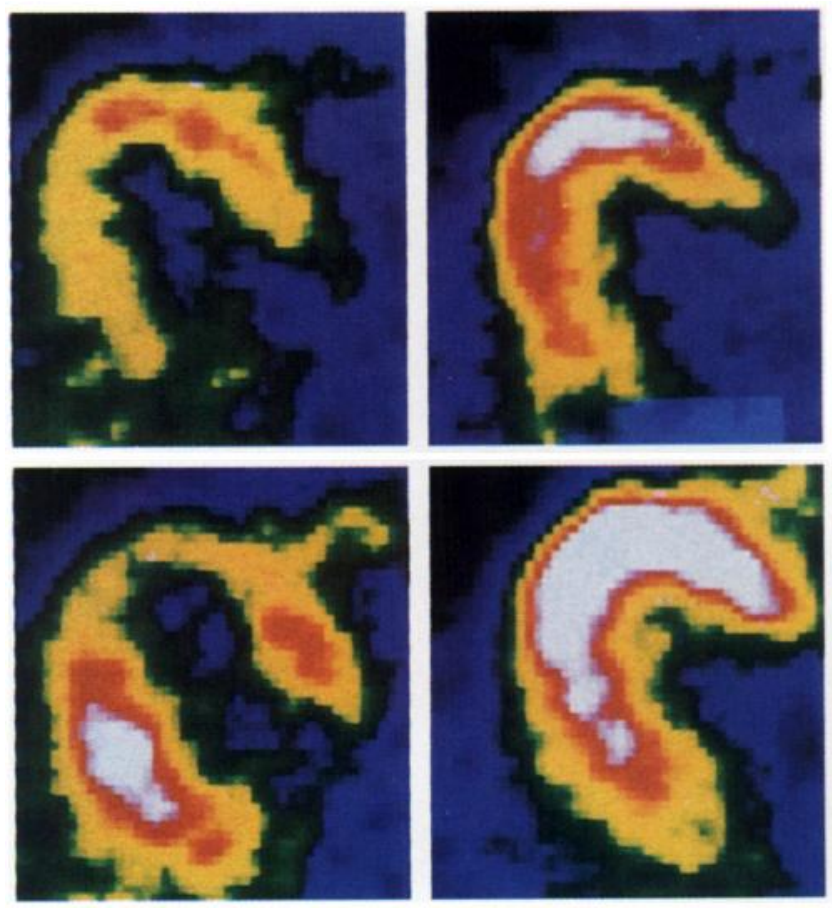

\section{FIGURE 1}

Positron images of $\left[{ }^{13} \mathrm{~N}\right]$ ammonia at the mid-ventricular level in Patient 4 before (left) and after successful angioplasty of a left anterior descending coronary artery stenosis (right). Resting images are at the top and dipyridamole-handgrip stress images are at the bottom. The improvement in the anatomic characteristics of the stenosis were accompanied by an increase in relative myocardial perfusion reserve $(0.75$ to 1.00$)$ indicating a good physiological effect. angioplasty. In all 11 patients, changes in relative myocardial perfusion reserve by positron tomography were consistent with changes in percent narrowing and arteriographically determined coronary flow reserve. In contrast, changes in absolute minimal cross-sectional lumen area did not correlate closely with changes in relative myocardial perfusion reserve. The lack of correlation with changes in absolute cross-sectional lumen area can probably be attributed to the fact that the physiological effect of a given absolute luminal area will depend on the original size of the artery at the location of the stenosis.

\section{Comparison to Other Approaches for Assessment of PTCA}

Several other approaches have been used to evaluate the functional effects of coronary angioplasty. Ganz et al. have suggested that provocation of a high transstenotic pressure gradient by intracoronary contrast injection is a useful measure of the significance of moderate stenoses and resolution of these induced gradients indicates successful angioplasty (13). However, this approach may be limited since the $2.0 \mathrm{~F}$ catheter does not cross the stenosis in as many as $20 \%$ of patients. Use of smaller bore catheters is associated with pressure damping and may impinge on the stenotic lumen significantly, thereby worsening the gradient independent of stenosis geometry.

O'Neill et al. have proposed that coronary vasodilatory reserve of 1.2 , measured using a digital radiographic technique, can be used to determine whether angioplasty is successful (19). However, follow-up stud- ies are not practical since repeat catheterization is required.

Exercise-redistribution thallium-201 scintigraphy has also been used to evaluate patients following PTCA. Several studies have reported that perfusion defects present before angioplasty resolve after successful angioplasty $(14,15)$. During follow-up periods up to 18 mo, Wijins et al. found that $74 \%$ of patients with thallium perfusion defects that filled in at redistribution had re-stenosis at repeat arteriography (17).

Although the physical decay properties of positron emitters are superior to single photon emitters, positron imaging has not been routinely used because of the cost involved in setting up a positron imaging facility. However, the recent development of $a^{82} \mathrm{Rb}$ generator allows positron perfusion imaging without the need for either a cyclotron or radiochemist $(10,20)$.

There are potential sources of error in the assessment of perfusion reserve with PET that must be considered. In animal experiments, uptake of $\left[{ }^{13} \mathrm{~N}\right]$ ammonia and ${ }^{82} \mathbf{R b}$ is linearly related to perfusion to flows up to three times resting flow (4-7). Higher flows are underestimated since uptake of tracer plateaus due to flow dependent decreases in the extraction fraction of the tracer. Differences in uptake could be due to differences in extraction between $\left[{ }^{13} \mathrm{~N}\right]$ ammonia and ${ }^{82} \mathrm{Rb}$. Despite this potential limitation, the approach can detect a $>50 \%$ diameter narrowing $(10,21)$. As with all radionuclide imaging studies, partial volume errors can also affect measured activity (12). In the current study, these potential errors were minimized by using the same region of interest at rest and after dipyridamole-handgrip stress. 


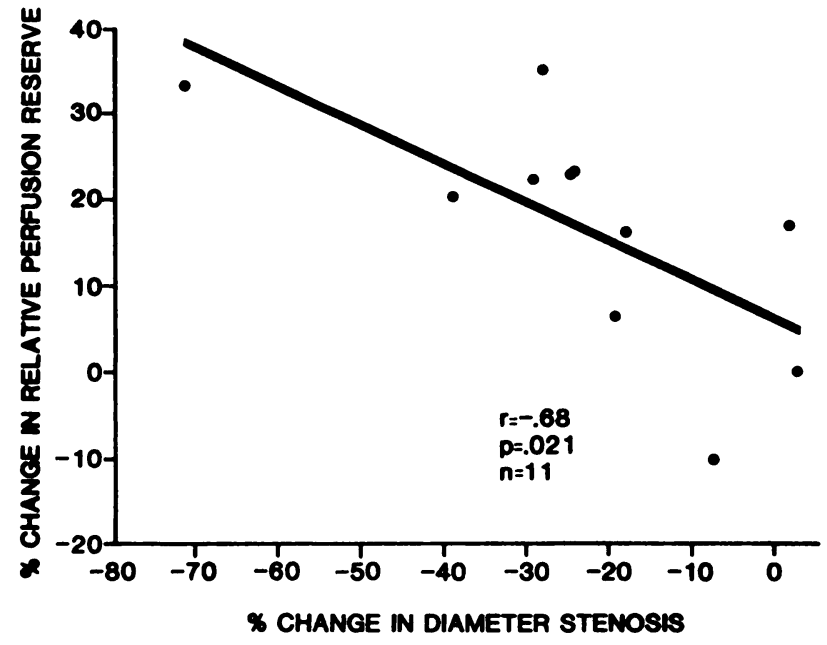

FIGURE 2

Relation between changes in percent diameter narrowing and changes in relative myocardial perfusion reserve.

The current study demonstrates a good agreement between changes in relative myocardial perfusion reserve with PET and changes in coronary anatomy after PTCA. It is difficult to compare the results of this study with those performed with thallium-201 because of several important differences in the way that the data in this study was obtained. First, all anatomic measures were derived using quantitative coronary arteriography. In other studies to date, visual estimation of percent narrowing represented the "gold standard". However, the large degree of variability in these qualitative assessments is a major limitation. Another difference is that the dipyridamole plus handgrip stress was used in this study rather than symptom limited exercise. This type of stress has the advantage of being able to produce regional disparities in perfusion with little effort on the

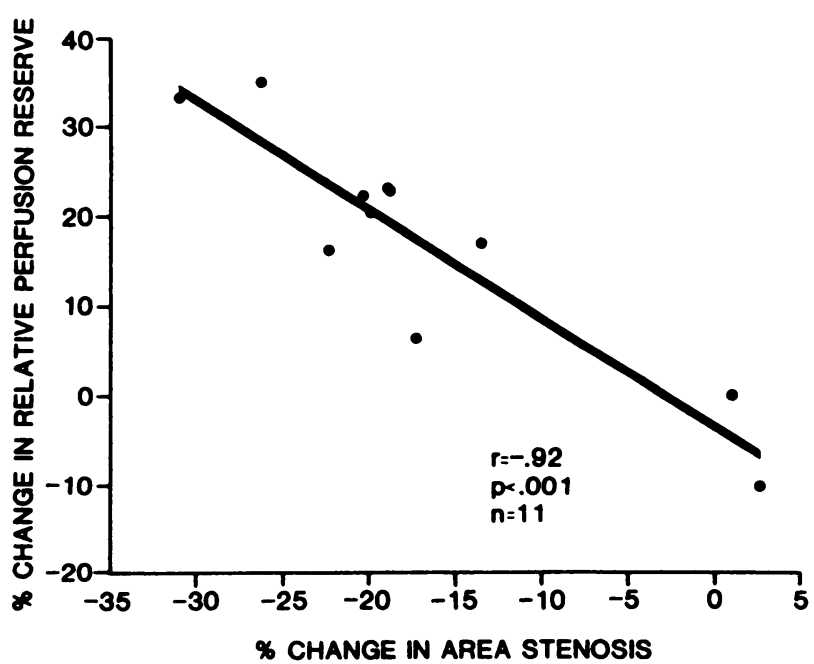

FIGURE 3

Relation between changes in percent area narrowing and changes in relative myocardial pertusion reserve.

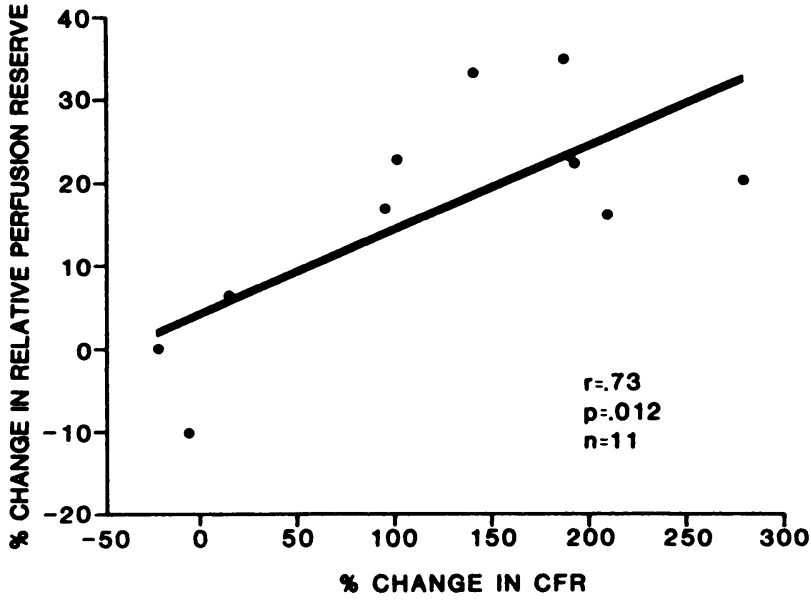

FIGURE 4

Relation between changes in angiographic coronary stenosis flow reserve and changes in relative myocardial perfusion reserve.

part of the patient. Thus, patients with mechanical limitations to exercise, obesity, or lack of motivation to exert themselves are minimized. The only limitations with dipyridamole is that there may be some variability in the amount of hyperemia that is produced in a given patient. Finally, the most important difference is that the perfusion data presented in this study was based on quantification of attenuation corrected regional activity. In contrast, thallium activity was either visually assessed or "quantified" without an attenuation correction.

\section{CONCLUSIONS}

The current study demonstrates the feasibility of positron imaging for following the physiologic effects of coronary angioplasty. Improvements in relative myocardial perfusion reserve by positron tomography correlate with increases in coronary stenosis flow reserve and reductions in percent narrowing. Additional studies are necessary to test whether serial follow-up studies allow early recognition of restenosis and to determine if these results are applicable to patients with multivessel disease.

\section{NOTES}

- Searle-Siemens Medical Systems, Inc., Iselin, NJ.

+ (Siemens Opti 150/40/72c) Searle-Siemens Medical Systems, Inc., Iselin, NJ.

\section{ACKNOWLEDGMENTS}

The authors wish to acknowledge the contribution of Mary Haynie, RN, Richard Holmes, Salma Marani, Mark Franceschini, Leonard A. Bolomey, Ross Hartz, Dan Burgess, Mark 
Wilson, Wai-Hoi Wong, PhD, Timothy J. Tewson, PhD, Marc Berridge, $\mathrm{PhD}$, and the technical staff of the Hermann Hospital cardiac catheterization laboratory, without whom this study would not have been possible. The authors also thank Shirley Mickelsen for her secretarial assistance.

This work was carried out as a joint collaborative research project with the Clayton Foundation for Research and was supported in part by Grants RO1HL26862 and RO1HL26885 from the National Institutes of Health, Bethesda, Maryland, Grant DE-FG05-84ER60210 from the Department of Energy, Washington, D.C. and a Grant-in-Aid from the American Heart Association, Dallas, Texas.

\section{REFERENCES}

1. Kirkeeide RL, Gould KL, Parsel L. Assessment of coronary stenoses by myocardial perfusion imaging during pharmacologic coronary vasodilation. VII. Validation of coronary flow reserve as a single integrated functional measure of stenosis severity reflecting all its geometric dimensions. J Am Coll Cardiol 1986; 7:103-113.

2. Harrison DG, White CW, Hiratzka LF, et al. The value of lesion cross-sectional area determined by quantitative coronary angiography in assessing the physiologic significance of proximal left anterior descending coronary arterial stenoses. Circulation 1984; 69:1111-1119.

3. Goldstein RA, Mullani NA, Gould KL. Quantitative myocardial imaging with positron emitters. In: Yu PN, Goodwin JF, eds. Progress in cardiology. Vol. 12. Philadelphia: Lea and Febiger, 1983: 147-191.

4. Mullani NA, Goldstein RA, Gould KL, et al. Myocardial perfusion with rubidium-82. I. Measurement of extraction fraction and flow with external detectors. $J$ Nucl Med 1983; 24:898-906.

5. Goldstein RA, Mullani NA, Fisher DJ, et al. Myocardial perfusion with rubidium-82. II. The effects of metabolic and pharmacologic interventions. $J$ Nucl Med 1983; 24:907-915

6. Schelbert HR, Phelps ME, Hoffman, EJ, et al. Regional myocardial perfusion assessed with $\mathrm{N}-13$ labeled ammonia and positron emission computerized axial tomography. Am J Cardiol 1979; 43:209-218.

7. Shah A, Schelbert HR, Schwaiger M, et al. Measurement of regional myocardial blood flow with $\mathrm{N}-13$ ammonia and positron emission tomography in intact dogs. J Am Coll Cardiol 1985; 5:92-100.

8. Gould KL, Kelley KO, Bolson EL. Experimental validation of quantitative coronary arteriography for determining pressure-flow characteristics of coronary stenoses. Circulation 1982; 66:930-937.

9. Kirkeeide RL, Fung P, Smalling RW, et al. Automated evaluation of vessel diameter from arteriograms. New York: Computers in Cardiology, Proceedings of IEEE Computer Society, 1982: 215-218.

10. Gould KL, Goldstein RA, Mullani N, et al. Noninvasive assessment of coronary stenoses by myocardial perfusion imaging during pharmacologic coronary vasodilation. VIII. Clinical feasibility of positron cardiac imaging without a cyclotron using generatorproduced rubidium-82. $\mathrm{J} \mathrm{Am}$ Coll Cardiol 1986; 7:775-789.

11. Mullani NA. Myocardial perfusion with rubidium-82: III. Theory relating severity of coronary stenosis to perfusion deficit. J Nucl Med 1984; 25:1190-1196.

12. Kent KM, Bentivoglid LG, Block PC, et al. Percutaneous transluminal coronary angioplasty: report from the registry of the National Heart, Lung and Blood Institute. Am J Cardiol 1982; 49:201 1-2020.

13. Ganz P, Abben R, Friedman PL, et al. Usefulness of transstenotic coronary pressure gradient measurements during diagnostic catheterization. Am J Cardiol 1985; 55:910-914.

14. Okada RD, Lim YL, Boucher CA, et al. Clinical, angiographic, hemodynamic, perfusional and functional changes after one-vessel left anterior descending cơronary angioplasty. Am J Cardiol 1985; 55:347356.

15. Hirzel HO, Nuesch K, Gruentzig AR, et al. Short and Long-term changes in myocardial perfusion after percutaneous transluminal coronary angioplasty assessed by thallium-201 exercise scintigraphy. Circulation 1981; 63:5.

16. Kalff V, Kelly MJ, Soward A, et al. Assessment of hemodynamic significance of isolated stenoses of the left anterior descending coronary artery using thallium-201 myocardial scintigraphy. Am J Cardiol 1985; 55:342-346.

17. Wijns W, Serruys PW, Reiber JHC, et al. Early detection of restenosis after successful percutaneous transluminal coronary angioplasty by exercise-redistribution thallium scintigraphy. Am $J$ Cardiol 1985; 55:357-361.

18. Wahr DW, Ports TA, Botvinick EH, et al. The effects of coronary angioplasty and reperfusion on distribution of myocardial flow. Circulation 1985; 72:2, 334 343.

19. O'Neill WW, Walton JA, Bates ER, et al. Criteria for successful coronary angioplasty as assessed by alterations in coronary vasodilatory reserve. $\mathrm{J} \mathrm{Am} \mathrm{Coll}$ Cardiol 1984; 3:6, 1382-1390.

20. Goldstein RA, Mullani NA, Wong W-H, et al. Positron imaging of myocardial infarction with rubidium82. J Nucl Med 1986; 27:1824-1829.

21. Goldstein RA, Kirkeeide R, Demer L, et al. Relation between geometric dimensions of coronary artery stenoses and myocardial perfusion reserve in man. $J$ Clin Invest 1987; 79:1473-1478. 